\title{
CONSOLIDATION DE LA VASE SOUS CONTRAINTES VARIABLES.
}

\author{
M. SANCHEZ ; A. GROVEL \\ Laboratoire de Mécanique et de Géomécanique de Nantes \\ Faculté des Sciences et des Techniques \\ 2. rue de la Houssinière, 44072 NANTES CEDEX
}

\begin{abstract}
:
The cohesive sediment dynamics must take into account the consolidation processes which brings dry bed density variability in time.

In this paper, a state-of-the-art concerning consolidation models commonly used in sedimentary dynamics is given, as well as, a review of the soils mechanics consolidation theories. The boundary between sedimentation and consolidation is defined, and similitude laws correlating prototype and laboratory data are presented.

Based on theoretical aspect, a consolidation model is developed. In this model, dry density $C$, characterize material state. The permeability and the material behaviour are only depending on the dry density. The material behaviour is simulated by a rigid-plastic model.

Consolidation tests of a mud (from the estuary of the Loire river) are carried out into laboratory. Tests results are used to adjust and check the validity of the proposed model.
\end{abstract}

\section{INTRODUCTION.}

La dynamique sédimentaire des matériaux en milieu aqueux, comporte les phénomènes d'érosion. de transport et de dépôt. L'étude de ces phénomènes sédimentaires montre que le comportement des matérioux est différent selon la dimension de leurs particules élémentaires. Les particules de dimension supérieure à $0.2 \mathrm{~mm}$ forment les matériaux granulaires. Ces particules conservent leur individualité en tout moment; la condition critique d'érosion vient de l'équilibre entre la force de traction et le frottement intergranulaire, la vitesse critique d'érosion étant essentiellement fonction de la dimension des particules pour une nature donnée de matériau.

Les particules de dimension inférieure à $0.2 \mathrm{~mm}$ forment les matériaux cohésifs. La résistance à l'érosion est fonction de la grandeur de la cohésion ou rigidité initiale des matériaux du fond, ainsi, à partir d'essais réalisés en canal au Laboratoire Central d'Hydraulique de France, Migniot (9) propose la formule suivante: 


$$
\begin{aligned}
u *_{c} & =0,013 \tau_{y}^{0,25} \text { pour: } \tau_{y}<3,00 \mathrm{~Pa} \\
u *_{c} & =0,009 \tau_{y}^{0,50} \text { pour: } \tau_{y}>3,00 \mathrm{~Pa} \\
\text { ovec: } \quad u *_{c} & =\sqrt{\tau_{0 c} / \rho_{0}}
\end{aligned}
$$

où : $\tau_{y}$, est la rigidité initiale du matériau $(P a) ; \tau_{\circ}$, est la tension de cisaillement critique d'érosion (Pa) : po, la masse volumique du fluide $\left(\mathrm{kg} \mathrm{m}^{-3}\right)$; e+ $\mathrm{u}^{*} \mathrm{c}$, la vitesse de cisaillement critique d'érosion $\left(\mathrm{m} \mathrm{s}^{-1}\right)$.

Des mesures réalisées au Laboratoire Centrale d'Hydraulique de France (LCHF) utilisant un viscosimètre à cylindres coaxiaux, de type "Brookfield", sur un grand nombre des vases ef des boues d'origine très diverses (marine. estuarienne, fluviatile, lacustre, minérale, et artificielle) permettent de déterminer la rigidité initiale en utilisant le modèle théologique de Bingham (Migniot (9)). Les résultats observés au LCHF, montrent que la rigidité initiale et la concentration en matières sèches $\mathrm{C}$, sont reliées par une loi de puissance de la forme:

$$
\tau_{y}=b C^{6}
$$

où: $b$, est un coefficient propre à chaque matériau, qui dépend de la nature des composants sédimentaires et du milieu aqueux. Le tableau i donne les valeurs du coefficient b pour différentes vases plastiques du littoral français étudiées au LCHF.

\begin{tabular}{|c|c|c|c|c|c|c|c|c|c|}
\hline Origine & Loire & $\begin{array}{c}\text { Ome } \\
\text { Ouistreham }\end{array}$ & Dunkerque & $\begin{array}{c}\text { La } \\
\text { Vilaine }\end{array}$ & $\begin{array}{c}\text { Seine } \\
\text { Roven }\end{array}$ & Gironde & $\begin{array}{c}\text { Le } \\
\text { Havre }\end{array}$ & Dives & $\begin{array}{c}\text { Mt. St. } \\
\text { Michel }\end{array}$ \\
\hline $\mathrm{b} / 10^{-5}$ & 21 & 11,5 & 9 & 6 & 4 & 2,4 & 0,92 & 0,36 & 0,26 \\
\hline
\end{tabular}

Tableau 1.- Valeur du coefficient b de l'équation (2). pour des vases plastiques du littoral français (d'après Migniot (9)).

Dans cette formule empirique, on observe que la rigidité initiale des matériaux (et donc, la tension de cisaillement critique d'érosion) augmente très fortement avec la concentration en matières sèches. D'autre part, la concentration en matières sèches dans un dépòt est essentiellement variable dans le temps par suite de la consolidation. La consolidation, est alors, un phénomène sédimentologique complémentaire de ceux mentionnés précédemment. 


\section{RAPPEL SUR LES MODELES DE CONSOLIDATION UTILISES DANS L'ETUDE DE LA DYNAMIQUE SEDIMENTAIRE .}

Un grand nombre d'essais de tassement des vases ont été réalisés au LCHF, en utilisant des tubes transparents de $0,10 \mathrm{~m}$ de diamètre. Ces essais ont permis d'étudier l'influence dans le tassement des paramètres suivants : hauteur initiale du dépôt; concentration initiale du dépôt : nature du milieu aqueux ; nature de la vase.

L'analyse descriptive des résultats du LCHF, vaut pratiquement pour toutes les vases (Lebreton (7)). La courbe donnant la concentration moyenne du culot de tassement en fonction du temps permet de distinguer plusieurs phases successives (Fig. 1):

1.- Le temps de floculation de quelques minutes en moyenne.

2.- La chute de flocons à vitesse entravée.

3.- La première phase de consolidation avec écrasement des flocons.

4.- La seconde phase dans laquelle l'eau est évacuée plus lentement par porosité. Des puits de drainage préférentiel peuvent apparaître.

5.- La troisième phase de consolidation, très lente, qui correspona a un arrangement de la structure du dépót et à la perte d'eau des couches adsorbées.

Les deux premières phases correspondent à un phénomène de sédimentation. à partir de la troisième phase les flocons se touchent et forment un réseau continu tridimensionnel ef il n'y a plus de décantation, mais consolidation. Les trois phases de consolidation précédentes, peuvent être décrites par une loi reliant la concentration moyenne du dépōt $\overline{\bar{C}}$, au temps t.

$$
\overline{\mathrm{C}}=\alpha \log _{10} \mathrm{t}+\beta
$$

où : $\alpha$, est un facteur qui dépend du complexe sol-eau; et $\beta$. un facteur lié au milieu aqueux.

La mesure de la concentration à l'intérieur du culot révèle également un gradient entre la surface et le fond. Après un temps de consolidation semi-infini. la loi de la variation de la concentration avec la profondeur est:

$$
\overline{\mathrm{C}}=\mathrm{C}_{\mathrm{s}}+\mathrm{n}_{1} \log _{10} \mathrm{~h}
$$

où : Cs, est la concentration à la surface du dépôt $\left(\mathrm{kg} \mathrm{m}^{-3}\right)$; h la profondeur en $\mathrm{cm}(\mathrm{h} \geq 1 \mathrm{~cm})$ : et $\mathrm{n} 1$. un paramètre dépendant de la nature du complexe soleau, pour les vases marines étudiées au LCHF, les valeurs de ni sont comprises entre 50 et 80 . 
Plus récemment, Le Hir et al (8). d'après une revue des mesures de consolidation effectuée par Dixit (4) (d'après Hayter(6)), proposent un modèle empirique sous la forme d'une équation différentielle relative à la concentration en matières sèches (Fig. 2).

$$
\frac{\partial \bar{C}}{\partial t}=\alpha_{0}\left(\bar{C}_{\infty}-\bar{C}\right)
$$

où: $\bar{C}_{\infty}$, est la concentration moyenne finale du dépôt: et $\alpha_{0}$, un facteur dépendant du complexe sol-eau.

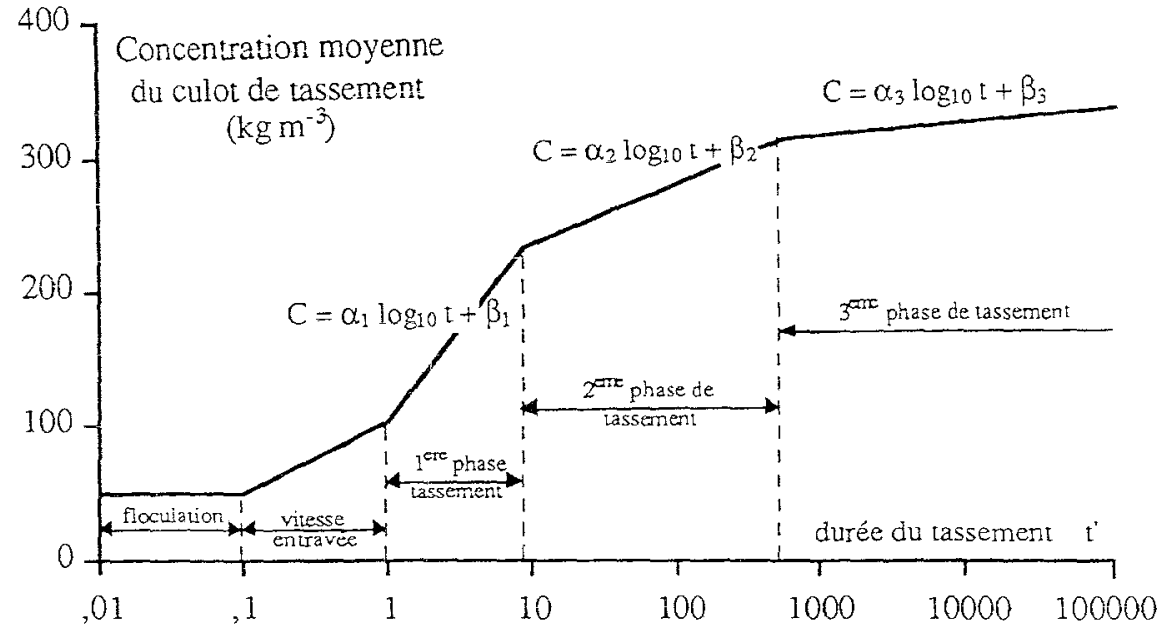

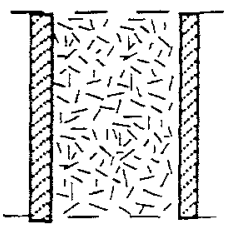

particules défloculées

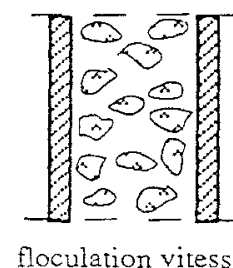

floculation vitesse de chute entravée

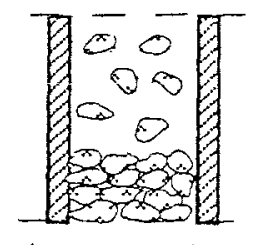

écrassement des

flocons élimination

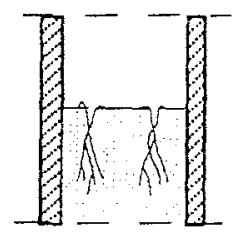

évacuation des eaux élimination des eaux par porosité

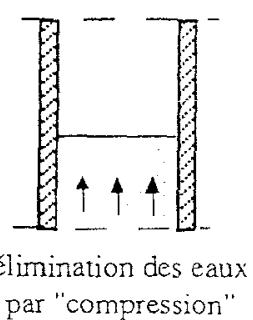

Fig. 1.- Variation du tassement de la vase en fonction du temps (d'après le LCHF) (réf. Lebreton (7)).

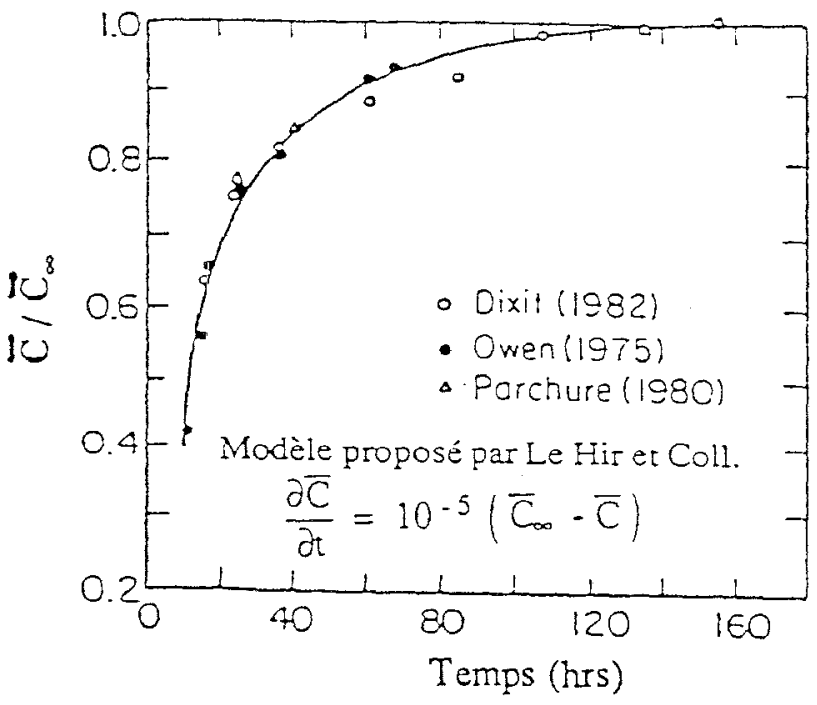

Fig. 2.- Revue des mesures de consolidation

(d'après Dixit (4), réf. Hayter (6)). 


\section{3.- FRONTIERE ENTRE LA SEDIMENTATION ET LA CONSOLIDATION.}

La frontière entre les phénomènes de sédimentation et de consolidation correspond à une concentration de la vase que nous désignons dans ce travail par Co - concentration la plus faible permettant la formation d'une structure à réseau continu tridimensionnel en l'absence des contraintes externes (Sanchez et Grovel (10)) - La concentration Co dépend essentiellement du diamètre des particules et de l'action électrolytique du milieu aqueux, c'est-à-dire, de la structure plus ou moins lâche des flocons de la suspension avant le dépôt. Les valeurs de co sont donc, très différentes selon le matériau étudié.

Une série d'essais de dépôt réalisés avec de la vase de Trentemoult (estuaire de la Loire) nous a permis de déterminer une valeur de co égale à 108 $\mathrm{kg} \mathrm{m}^{-3}$

Carrier et al (2) à partir d'essais de consolidation réalisés en laboratoire sur des boues d'origine très diverses, montrent que la limite entre les phénomènes de sédimentation et de consolidation correspond à une teneur en eau égale à 7 fois la limite de liquidité du matériau. La limite de liquidité de la vase que nous avons étudiée en laboratoire est de $150 \%$, ce qui correspond selon Carrier à une concentration Co de $91,8 \mathrm{~kg} \mathrm{~m}^{-3}$.

\section{4.- THEORIES "CLASSIQUES" DE CONSOLIDATION.}

\section{Modèle de Teraghi.}

La théorie unidimensionnelle de consolidation "classique" développée par Terzaghi et Peck (12), a été utilisée avec succès dans une grande quantité de problèmes de surconsolidation en mécanique de sols. Dans la plupart des problèmes pratiques, les sols ayant subi une consolidation préalable importante, les hypothèses introduites par Terzaghi dans sa théorie de consolidation sont valables, a savoir :

1.- le sol est homogène:

2.- le sol est complètement saturé:

3.- l'eau et les grains du sol sont incompressibles:

4.- l'écoulement de l'eau est vertical:

5.- validité de la loi de Darcy:

6.- le retard de la compression n'est dû qu'à la faible perméabilité du matériau:

7.- La contrainte de consolidation est appliquée instantanément:

8.- les déformations sont petites vis-à-vis de la hauteur initiale: 
9.- le sol a un comportement élastique linéaire :

10.- la perméabilité est constante dans le temps et dans l'espace :

11.- le coefficient de compressibilité volumétrique est constant :

12.- la vitesse de la phase solide est négligeable devant celle de la phase fluide.

A partir du principe de contrainte effective, de la loi d'écoulement de Darcy et de l'équation de continuité de la phase liquide, Terzaghi obtient l'équation générale de consolidation :

$$
\frac{\partial u}{\partial t}=C_{v} \frac{\partial^{2} u}{\partial z^{2}} \quad \text { avec: } C_{v}=\frac{k}{\rho_{0} g m_{v}} \quad \text { et } \quad m_{v}=\frac{d n}{d \sigma^{\prime}}
$$

où : $u$, est la surpression interstitielle (Pa) $C_{V}$, le coefficient de consolidation $\left(\mathrm{m}^{2} \mathrm{~s}^{-1}\right) ; \boldsymbol{k}$, le coefficient de perméabilité $\left(\mathrm{m} \mathrm{s}^{-1}\right)$; po, la masse volumique de la phase liquide du sol $\left(\mathrm{kg} \mathrm{m}^{-3}\right) ; \mathrm{g}$ l'accélération de la pesanteur $\left(\mathrm{m} \mathrm{s}^{-2}\right) ; \sigma^{\prime}$, la contrainte effective $(\mathrm{Pa})$; $\mathrm{n}$ la porosité du sol (définie pour un sol saturé comme étant le quotient du volume de la phase liquide par le volume totale du sob) : et $\mathrm{mv}$, le coefficient de compressibilité volumétrique $\left(\mathrm{Pa}^{-1}\right)$.

Pour interpréter les résultats on définit l'indice de consolidation local IC, ef le degré de consolidation du dépôt $U$.

$$
I C=\frac{u(z, 0)-u(z, t)}{u(z, 0)} \text { et } \quad U=1-\int_{0}^{\mathrm{H}} \mathrm{u}(\mathrm{z}, \mathrm{t}) \mathrm{dz} / \mathrm{f}_{0}^{\mathrm{H}} \mathrm{t}(\mathrm{z}, 0) \mathrm{dz}
$$

Les conditions aux limites correspondant à la consolidation sous poids propre, sur un fond imperméable, d'un dépôt d'épaisseur $H(m)$ sont: $u(0, t)=0$; $u(z, 0)=u(H, 0) z / H$ pour $0<z<H$; et $\partial u(H, t) / \partial z=0$ pour $\dagger>0$. La solution de l'équation ( 6 ) pour les conditions aux limites précédentes donne IC et $U$ par (Fig. 3 et Fig. 4)

$$
\begin{aligned}
& I C=1-\frac{H}{z} \sum_{m=0}^{\infty}(-1)^{m} \frac{2}{M^{2}} \sin \left(M \frac{Z}{H}\right) e^{-M^{2} T v} \\
& U=1-\sum_{m=0}^{\infty}(-1)^{m} \frac{4}{M^{3}} e^{-M^{2} T v}
\end{aligned}
$$

avec: $\quad M=\frac{(2 \mathrm{~m}+1) \pi}{2} \quad$ et $\quad T_{\mathrm{v}}=\frac{\mathrm{C}_{\mathrm{V}}}{\mathrm{H}^{2}} \mathrm{t}$ 


\section{Modèle de consolidation de Gibson.}

Un autre modèle de consolidation est celui de Gibson et al (5), lequel considère la non-linéarité de la perméabilité et de la dérivée de la contrainte effective par rapport à l'indice de vides $d \sigma$ '/de.

$\frac{\partial}{\partial z}\left[\frac{k(e)}{\rho_{0} g(1+e)} \frac{d \sigma^{\prime}}{d e} \frac{\partial e}{\partial z}\right]+\left[\frac{\rho_{s}-\rho_{0}}{\rho_{0}}\right] \frac{d}{d e}\left[\frac{k(e)}{1+e}\right] \frac{\partial e}{\partial z}=-\frac{\partial e}{\partial t}$ avec: $\left\{\begin{array}{l}k=f_{1}(e) \\ d \sigma^{\prime} / d e=f_{2}(e)\end{array}(10)\right.$

où : e, est l'indice de vides, défini pour les sols complètement saturés, comme étant le quotient du volume de la phase liquide par le volume de la phase solide.

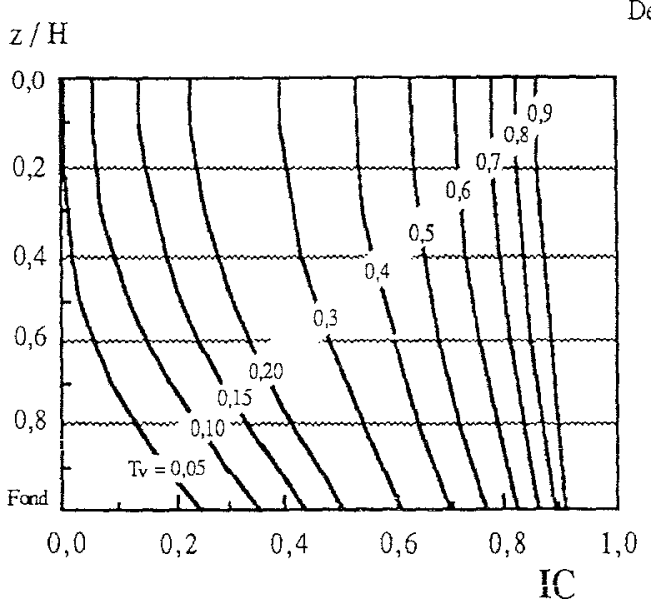

Fig. 3: Variation de I'indice de consolidation en fonction de la profondeur et de TV (d'après Tezaghi (12))
Degré de Consolidation

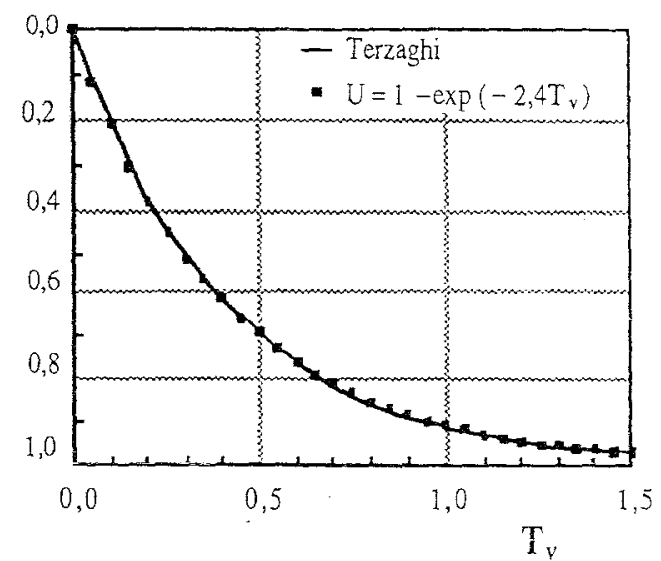

Fig. 4.-Variation du degré de consolidation en fonction de TV (d'après Terzaghi (12)).

\section{SIMILITUDE EN CONSOLIDATION.}

Si toutes les hypothèses introduites dans la théorie de consolidation de Teraghi sont justifiées et surtout si les déformations sont petites vis-à-vis de la hauteur initiale du dépôt, la variation de la concentration moyenne et celle du degré de consolidation sont liées par:

$$
\frac{\overline{\mathrm{C}}-\overline{\overline{C I}}}{\overline{\mathrm{C}}_{\infty}-\overline{\mathrm{CI}}}=\mathrm{U}
$$

où : $\overline{\mathrm{Cl}}$.étant la concentration initiale du dépôt. 
D'autre part, dans le cas d'un matériau consolidé sous poids propre, le degré de consolidation est approché par (Fig. 4):

$$
U=1-\exp \left(-2,4 T_{V}\right)
$$

En combinant ( 11 ) et ( 12 ), dérivant par rapport à $t$, et après rangement, on obtient :

$$
\frac{\partial \overline{\mathrm{C}}}{\partial \mathrm{t}}=\left(2,4 \frac{\mathrm{C}_{\mathrm{v}}}{\mathrm{H}^{2}}\right)\left(\overline{\mathrm{C}}_{\infty}-\overline{\mathrm{C}}\right)
$$

Nous observons, donc. que la formulation proposée par Le Hir et al (équation ( 5 )) se rapproche de la solution de Terzaghi pour le degré de consolidation si :

$$
\alpha_{0}=2,4 \mathrm{C}_{\mathrm{v}} / \mathrm{H}^{2}
$$

En général, lorsque les hypothèses de Terzaghi sont satisfaites, le degré de consolidation d'un dépôt d'épaisseur $H$, qui représente un prototype est donnée selonl'équation (9), par:

$$
\mathrm{U}=f_{\mathrm{o}}\left(\frac{\mathrm{C}_{\mathrm{v}} \mathrm{t}}{\mathrm{H}^{2}}\right)
$$

Dans les essais oedométriques, on reproduit sur le matériau du prototype les mêmes contraintes de consolidation, mais l'épaisseur du prototype est représentée par $\mathrm{Hm}<\mathrm{H}$. Si dans un essai oedométrique on observe un degré de consolidation $\mathrm{u}$ au temps $t \mathrm{~m}$, le temps $t$, pour avoir le même degré de consolidation dans le prototype est déterminé moyennant l'échelle de temps suivante:

$$
\mathrm{H}^{2} \mathrm{t}_{\mathrm{m}}=\mathrm{H}_{\mathrm{m}}^{2} \mathrm{t}
$$

Les essais oedométriques ne reproduisent ni les conditions de drainage ni la distribution triangulaire des contraintes à l'intérieur d'un dépôt se consolidant sur poids propre. Les études sur modèles réduits centrifugés permettent de pallier ce problème. Dans un modèle centrifugé. si $H$ est l'épaisseur correspondant au prototype. $\mathrm{Hm}$. l'épaisseur du modèle. l'accélération du modèle $\mathrm{Ng}$, nécessaire à reproduire les contraintes de consolidation est (Corté et Garnier (3)).

$$
\mathrm{Ng}=\mathrm{g} \frac{\mathrm{H}}{\mathrm{H}_{\mathrm{m}}}
$$


Le temps du modèle est prototype sont liés par:

$$
t=N^{2} t_{m}
$$

Balay et al (1) ont réalisé des essais dans la centrifugeuse du LCPC-Nantes (Corté et Garnier (3)), pour simuler la consolidation sous poids propre des vases de la Rochelle-Pallice. Ils reproduisent les résultats observés en laboratoire, en utilisant l'équation générale de la consolidation non-linéaire unidirectionnelle de Gibson (équation ( 10 )), avec $\mathbf{d} \sigma^{\prime} /$ de et $\boldsymbol{k}(e)$ donnés par les formulations proposées par Carrier et al (2) pour les argiles très molles, en fonction de l'indice de plasticité IP, à savoir :

$\frac{\mathrm{d} \sigma^{\prime}}{\mathrm{de}}=7,9510^{-5} \operatorname{IP}^{3,45} \mathrm{e}^{-3,45} \quad$ et $\quad \mathrm{k}(\mathrm{e})=1,74 \mathrm{IP}^{-4,29} \mathrm{e}^{4,29} /(1+\mathrm{e})(19)$

où : $\mathbf{d} \sigma^{\prime} / \mathrm{de} \mathrm{s}^{\prime}$ exprime ici en $\mathrm{kPa}$; et $\mathbf{k}(e)$ en $\mathrm{cm} \mathrm{s}^{-1}$.

\section{VERS UN MODELE THEORIQUE DE CONSOLIDATION DE VASES PEU CONCENTREES .}

Les modèles de consolidation de Terzaghi et de Gibson, considérant que le comportement du sol est élastique, ne s'adaptent pas à la modélisation de la consolidation des vases molles. Par conséquent, nous sommes amenés à revenir à la forme primitive des équations gouvernant la consolidation, afin de formuler un modèle qui s'adapte à l'étude de la consolidation sous poids propre des vases peu concentrées.

Le fait que le modèle de consolidation sera postérieurement introduit dans un modèle hydrosédimentologique amène à utiliser la concentration en matières sèches comme variable de l'état du matériau. Lorsque la masse volumique de la phase solide ps, est connue et que le matériau est complètement saturé, il y a une correspondance entre la concentration en matières sèches et la porosité où l'indice des vides.

$$
\mathrm{n}=1-\frac{\mathrm{C}}{\rho_{\mathrm{s}}} \quad ; \quad \mathrm{e}=\frac{\rho_{\mathrm{s}}}{\mathrm{C}}-1 \quad ; \quad \mathrm{n}=\frac{\mathrm{e}}{\mathrm{e}+1}
$$

Dans cette étude, le comportement de la vase est assimilé à un modèle rigideplastique, ainsi, lorsque l'on enlève une contrainte (lors des érosions par exemple) la concentration reste constante. L'augmentation de la concentration est possible seulement Iorsque la contrainte de consolidation est supérieure à la contrainte de consolidation préalable.

D'autre part, on considère que le comportement à la compressibilité de la vase est non-visqueux, c'est à dire que la compression de la vase est indépendante de la vitesse d'application de la contrainte effective (par dissipation de surpression interstitielle). Ainsi. I'hypothèse 6 de Teraghi est valable. 
En plus des 6 premières hypothèses mentionnées dans le développement de Terzaghi, nous introduisons les hypothèses de travail suivantes:

7 - le matériau a un comportement rigide-plastique, ne dépendant que de la concentration C:

8- la perméabilité est fonction seule de la concentration:

9 - la base du dépôt est imperméable

1. Développement de notre modèle.

\subsection{Permécibilité .}

Lorsque la vitesse de la phase solide n'est pas négligeable devant celle de la phase fluide, la loi de Darcy est donnée par :

$$
n\left(v_{f}-v_{s}\right)=-k i \quad \text { avec }: \quad i=\frac{1}{\rho_{0} g} \frac{\partial u}{\partial z}
$$

où : $v f$, est la vitesse de leau interstitielle $\left(\mathrm{m} \mathrm{s}^{-1}\right)$; $v_{s}$, la vitesse de la phase solide $\left(\mathrm{m} \mathrm{s}^{-1}\right)$; i, le gradient hydraulique sans dimensions : et $k$, le coefficient de perméabilité $\left(\mathrm{m} \mathrm{s}^{-1}\right)$.

L'équation de continuité pour une colonne de dépôt est :

$$
\frac{\partial}{\partial z}\left[(1-n) v_{s}+n v_{f}\right]=0
$$

Si le fond est imperméable $\left(v_{f}=v_{s}=0\right.$ pour $\left.z=0\right)$, les équations $(20)$ et $(21)$ conduisent à :

$$
\mathrm{v}_{\mathrm{s}}=\mathrm{ki}
$$

Le coefficient $k$, en fonction de la concentration peut être approché d'après Tan et al (11) par:

$$
\log _{10} k=-a_{1} C-a_{2}
$$

En fonction de c / ps, l'équation précédente peut être mise sous la forme

$$
k=A_{1} \exp \left(-A_{2} C / p_{s}\right)
$$


7.2 Contrainte de consolidation.

La contrainte de consolidation sur un plan horizontal localisé en z, est égale au poids déjaugé de matériau au dessus de ce plan, par unité de surface, a savoir :

$$
\sigma_{z_{1}}=\left[1-\frac{\rho_{0}}{\rho_{s}}\right] g \int_{z_{1}}^{H} \mathrm{C} d z
$$

où: Hest le niveau de la surface du dépôt.

\subsection{Contrainte effective.}

Lors de la dissipation complète de la surpression interstitielle $\left(\sigma=\sigma^{\prime}\right)$, nous approchons la concentration finale de la vase $\mathrm{C}_{\infty}$, en fonction de la contrainte effective par:

$$
C_{\infty}=C_{0}+B_{1} \sigma^{\prime} B_{2}
$$

La contrainte de consolidation préalable $\sigma^{\prime} \mathrm{cp}$. d'un matériau ayant une concentration C > Co. peut être évaluée à partir de l'équation précédente.

$$
\sigma_{\mathrm{Cp}}^{\prime}=\left(\frac{\mathrm{C}-\mathrm{C}_{0}}{\mathrm{~B}_{1}}\right)^{1 / \mathrm{B}_{2}}
$$

La consolidation de la vase est possible seulement si la contrainte de consolidation est supérieure à la contrainte de consolidation préalable; donc, si les sollicitations sont très lentes, la contrainte effective (transmise grain à grain dans la phase solide) est :

$$
\sigma^{\prime}=\sigma_{c p}^{\prime} \quad \text { pour }: \sigma \geq \sigma_{c p}^{\prime}
$$

Si la contrainte de consolidation préalable est supérieure à la contrainte de consolidation (matériau surconsolidé), le comportement du matériau est assimilé à un modèle rigide, la contrainte de consolidation étant prise complètement par la phase solide, dans ce cas nous avons:

$$
\sigma^{\prime}=\sigma \quad \text { pour }: \sigma<\sigma_{\mathrm{cp}}^{\prime}
$$

D'autre part, le principe de contrainte effective implique qu'en un temps:

$$
u=\sigma-\sigma^{\prime}
$$


1.4 Continuité.

La condition de continuité de la phase solide, en coordonnées d'Euler est :

$$
\frac{\partial C}{\partial t}+\frac{\partial C v_{s}}{\partial z}=0
$$

\subsection{Equation de consolidation pour des sols normalement consolidés.}

On dit qu'un sol est normalement consolidé, si en tout moment la contrainte de consolidation est supérieure ou égale à la contrainte de consolidation préalable. La plupart des matériaux n'étant pas soumis au phénomène d'érosion sont normalement consolidés. Dans ce cas spécifique. les équations (22), (24),(29), et ( 30 ) conduisent à:

$$
\frac{\partial C}{\partial t}=\frac{\partial\left[v_{s s} C\right]}{\partial z}+\frac{\partial\left[C_{c} \partial C / \partial z\right]}{\partial z}
$$

avec: $\quad C_{c}=k \frac{1}{\rho_{0} g} C \frac{d \sigma}{d C} \quad$ et $\quad v_{s s}=\frac{k}{\rho_{0}}\left(1-\frac{\rho_{0}}{\rho_{s}}\right) C$.

\section{ETUDE DE LA CONSOLIDATION EN LABORATOIRE .}

Nous avons suivi en laboratoire, pendant 100 jours, l'évolution de 7 colonnes de tassement, contenant une vase provenant de l'estuaire de la Loire, se consolidant à une température constante de $21^{\circ} \mathrm{C}$. dans un milieu aque ux ayant une salinité de $20 \%$.

Les conditions initiales de nos colonnes de consolidation sont données dans la Tableau 2. La mesure des hauteurs finales (après 100 jours de consolidation), nous a permis -sous l'hypothèse que la surpression interstitielle était complètement dissipée- d'évaluer les paramètres B1 et B2 de l'équation (25).(Fig. 5).

A partir de notre développement théorique, nous avons construit un modèle numérique, qui est calé en faisant varier les paramètres $A_{1}$ et $A_{2}$ de notre loi de perméabilité (équation (23)) : le calage simultané de l'ensemble de colonnes nous conduit à :

$$
A_{1}=5 \times 10^{-3} \mathrm{~ms}^{-1} \text { et } A_{2}=110 \text { valable pour: } 108<C<332 \mathrm{~kg} \mathrm{~m}^{-3}
$$


La variation avec la concentration de $k, C d \sigma^{\prime} / d c$, et $C c$, pour les paramètres

$A_{1}, A_{2}, B_{1}$ et $B_{2}$, correspondant au calage simultané de toutes les colonnes de consolidation étudiées en laboratoire, est montrée dans la figure 6 .

L'évolution de l'épaisseur des dépôts avec le temps est montrée dans la figure 7. L'évolution de la concentration avec le temps et la profondeur pour la colonne de hauteur initiale de $1,500 \mathrm{~m}$ est montrée dans la figure 8.

\begin{tabular}{|c|ccccccc|}
\hline No. de colonne & 1 & 2 & 3 & 4 & 5 & 6 & 7 \\
\hline Hauteur initiale $(\mathrm{m})$ & 0,150 & 0,300 & 0,506 & 0,753 & 1,045 & 1,500 & 2,000 \\
Concentration initiale $\left(\mathrm{kg} \mathrm{m}^{-3}\right)$ & 108,0 & 108,0 & 108,0 & 128,8 & 128,8 & 108,0 & 108,0 \\
\hline
\end{tabular}

Tableau 2.: Conditions initiales dans nos colonnes de consolidation étudiées en laboratoire.

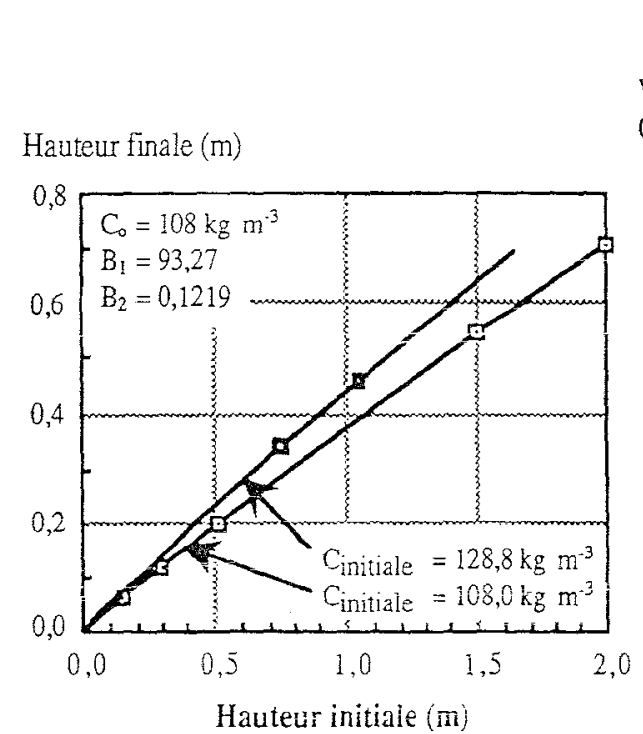

Fig. 5. : Calage de la loi de compressibilité de la vase (équation (25)), à partir des niveaux initiaux et finaux dans nos colonnes de consolidation.
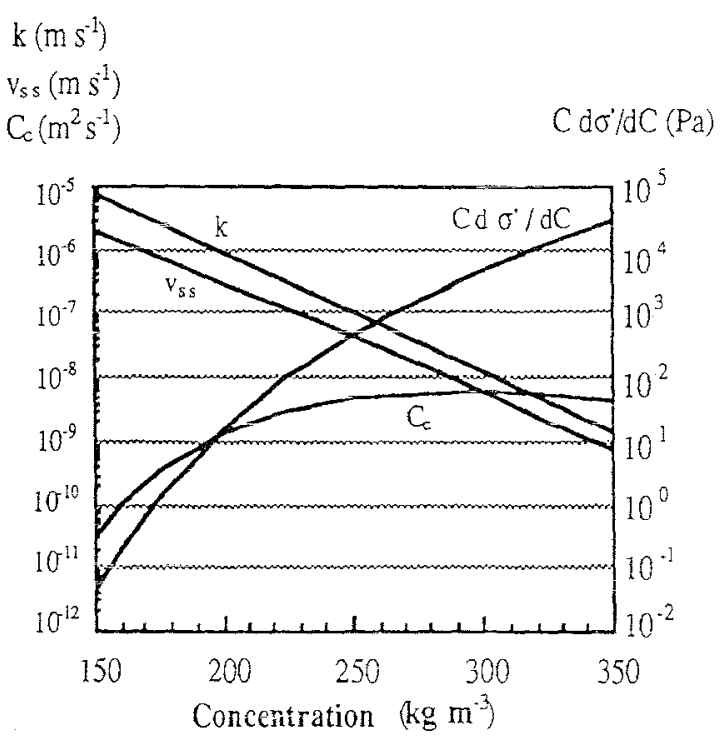

Fig. 6.- Variation de différents paramètres intervenant dans notre modèle de consolidation. avec la concentration 


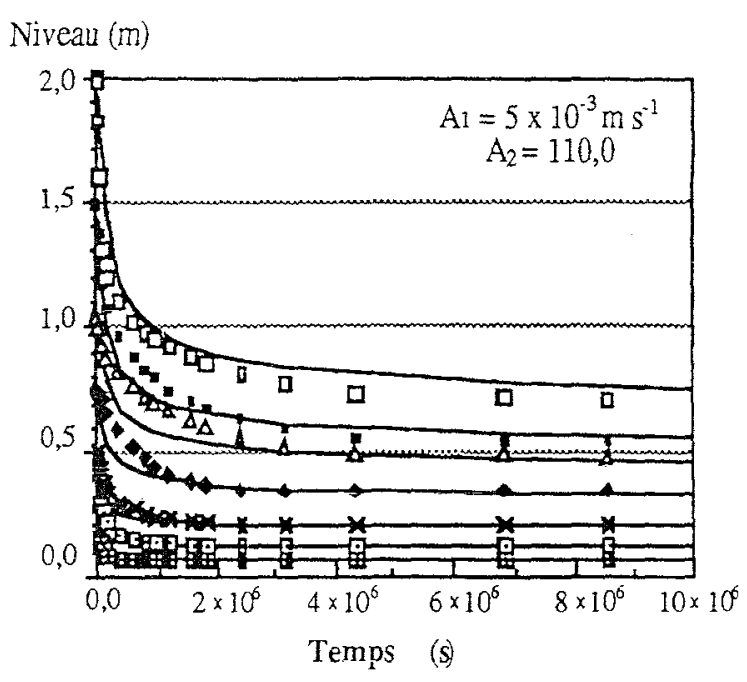

Fig.7. : Evolution de l'épaisseur des dépôts avec le temps (à partir de notre modèle).

\section{CONCLUSIONS}

1.- D'après la condition de similitude donnée par l'équation (16), un dépôt de vase atteint sa concentration finale d'autant plus rapidement que l'épaisseur initiale est faible.

2.- Le retard de la compression étant dú exclusivement à la faible perméabilité du matériau, la consolidation est d'autant plus rapide que la perméabilité est forte, ou d'après les équations (19) et (23) (Fig. 6), que la concentration est faible.

3.- Notre modèle de consolidation, formé par les équations (23) à (30), contient 5 paramètres qui sont propres à chaque matériqu : 2 paramètres pour la loi de perméabilité $\left(A_{1}\right.$ et $\left.A_{2}\right)$, et 3 pour la loi de compressibilité du matériau (Co. $\mathrm{B}_{1}$ et $\left.\mathrm{B}_{2}\right)$. La seule variable indépendante est la concentration.

4.- La variabilité des contraintes de consolidation, associée aux phénomènes d'érosion et de sédimentation, est prise en compte : lorsque la contrainte de consolidation est supérieure à la contrainte de consolidation préalable, le comportement du matériau est simulé par un modèle plastique : dans le cas contraire, nous considérons que le matériau est rigide.

5.- Le calage de notre modèle, colonne par colonne, nous montre que pour une même concentration, le coefficient de perméabilité semble être d'une grandeur d'autant plus importante que la hauteur initiale du dépôt est plus élevée.

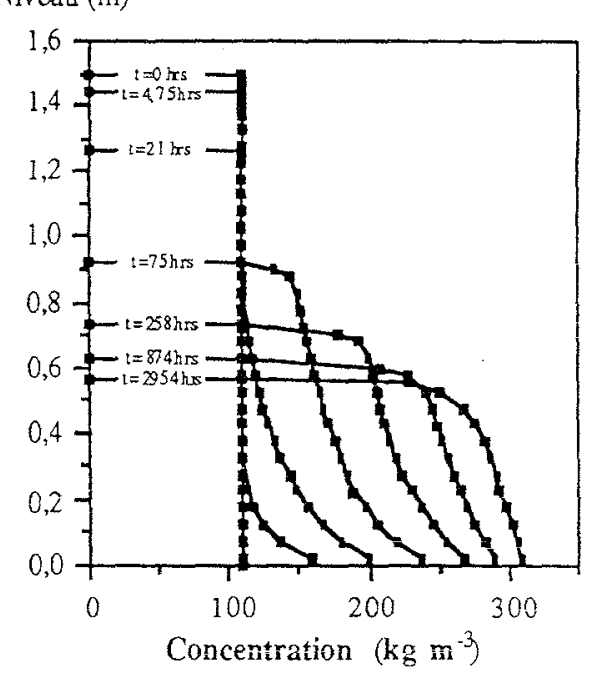

Fig. 8. : Evolution de la concentration dans le temps. à l'intérieur d'un dépôt (à partir de notre modèle). 
6.- D'après notre modèle et le modèle de Terzaghi, on observe que si le fond est imperméable, la consolidation de la vase est un phénomène qui commence par le fond des dépôts (Fig. 3 et Fig. 8)

7.- Un modèle empirique, permettant de simuler la consolidation de la vase en fonction de la profondeur et du temps, pourrait être développé à partir des équations suivantes:

$\frac{\mathrm{C}-\mathrm{CI}}{\mathrm{C}_{\infty}-\mathrm{CI}}=\mathrm{IC}=f_{\mathrm{a}}\left(\mathrm{T}_{\mathrm{v}}, \frac{\mathrm{z}}{\mathrm{H}}\right) ; \mathrm{C}_{\infty}=f_{\mathrm{b}}(\sigma) ;$ et $\frac{\partial \mathrm{C}}{\partial \mathrm{t}}=0$ pour : $\mathrm{C}_{\infty}(\sigma)<\mathrm{C}$

\section{BIBLIOGRAPHIE .}

(1) Balay, J., R. Berdat et L. Harfouche. "Etude en centrifugeuse de la consolidation sous poids propre de sols très lâches". Centrifuge 88 , edité par Corté, Balkema, Rotterdam, 1988.

(2) Carrier, W.D.. L.G. Bromwell and Frank Somogyi. "Design Capacity of Slurried Mineral Waste Ponds". Journal of Geotechnical Engineering, ASCE, vol. 109. No. 5, May, 1983, pp 699-716.

(3) Corté. J. F., et J. Garnier. "Une centrifugeuse pour la Recherche en Géotechnique". Bulletin de Liaison des Laboratoires des Ponts et Chaussées. No. 146, 1986, pp 5-28.

(4) Dixit, J.G.. "Resuspension Potential of Deposited Kaolinite Beds". M.S. Thesis, University of Florida. Gainesville. Florida, 1982.

(5) Gibson, R.E., G. L. England and M. J.L. Hussey. "The Theory of OneDimensional Consolidation of Saturated Clays, 1. Finite Nonlinear Consolidation of Thin Homogeneous Layers“. Geotechnique. Vol. 17, No. 3. 1967, pp $26 i-273$.

(6) Hayter, E.J.. "Estuarial Sediment Bed Model". Estuarine Cohesive Sediment Dynamics, edited by Mehta. Springer-Verlag. New York, 1986, pp326-359.

(7) Lebreton, J.C.. "Transport Solide". Mécanique des Fluides Appliquée, edité par Hug, Eyrolles, 1975, pp 453-594.

(8) Le Hir, P., P. Bassoullet, et J.L'Yavanac."New Developments about Mud Transport Models, Application to a Macrotidal Estuary". Proceedings of the International Symposium New Orleans, edited by S.S.Y. Wang. August 14-18. 1989, pp 94-99 
(9) Migniot, C.. "Tassement et Rhéologie des Vases", La Houille Blanche. No. I et 2, 1989 .

(10) Sanchez, M. et A. Grovel, "Etude des Phénomènes de Dépôt, de Consolidation et d'Erosion de la Crème de vase dans les Estuaires". Premières Journées Inter-Universitaires Génie Civil-Génie Côtier, Le Havre. 1990, pp 275-289.

(11) Tan, T.S., K.Y. Yong, E.C. Leong and S.L. Lee. "Sedimentation of Clayey Slurry". Journal of Geotechnical Engineering, Vol. 116. No. 6. June 1990, pp 885898.

(12) Terzaghi, K. and R.B. Peck. Mécanique des Sols Appliquée, Ed. Dunod. Paris, 1961. 